\title{
El capital humano en empresas peruanas de generación eléctrica
}

Recibido: 18/11/11 Aceptado: 20/06/12

Néstor Santos Jiménez ${ }^{1}$

\begin{abstract}
RESUMEN
El capital humano es un recurso valioso, cuya gestión no solo implica una adecuada compensación económica por su labor, sino también hacerlo partícipe de los retos de la empresa, de modo que se sienta parte de ella y como tal adopte una actitud de compromiso con todos los grupos de interés de la organización. Según la información pública analizada del periodo 2007-2010, en las principales empresas de generación eléctrica de Perú destaca un esfuerzo importante en la gestión del capital humano, con beneficios sociales atractivos y competitivos, lo que contribuye a la eficiencia y la competitividad de dichas empresas.
\end{abstract}

Palabras Clave: capital humano, productividad, gastos de personal, eficiencia, utilidad neta.

\section{HUMAN CAPITAL IN POWER}

GENERATION PERUVIAN COMPANY

\section{ABSTRACT}

Human capital is a valuable resource whose management involves not only an adequate compensation for their work but also make him partaker of the challenges of the company, so you feel part of it and as such adopt an attitude of commitment to all stakeholders of the organization. According to public information analyzed for the period 20072010, the main electricity generating companies in Peru highlights a major effort in human capital management, with attractive and competitive benefits, contributing to efficiency and competitiveness of these companies.

Keywords: Human capital, productivity, personnel costs, efficiency, net income.

\section{INTRODUCCIÓN}

El capital humano conjuntamente con el capital físico (infraestructura), los procesos de operación y la información permiten a las empresas generar valor agregado, dando lugar a organizaciones competitivas y sostenibles en el tiempo. El capital o talento humano, usualmente denominado también recurso humano, trabajador, personal o mano de obra, requiere de un adecuado trato en la gestión de las empresas, sean estas privadas o públicas, productoras de bienes o prestadoras de servicios, pequeñas o grandes.

Gracias al capital humano, en las diversas etapas de un negocio, se crean y mejoran procesos, se genera y administra información y se diseña, construye, opera y mantiene la infraestructura de la empresa. El talento humano no es un simple factor de producción, sino que es un recurso valioso que para un determinado tipo de empresa puede resultar escaso y costoso captarlo, capacitarlo y conservarlo en la organización.

En términos económicos, cada trabajador responde por una determinada inversión de la empresa, no solo en la operación y mantenimiento de la infraestructura sino también en la actitud que adopta en el desenvolvimiento de sus actividades, de prever cambios posibles y proponer acciones para aprovechar oportunidades y/o plantear planes de contingencia para amortiguar los riesgos. Por esta razón, en una organización se requieren diversas competencias y un cuerpo directriz que fije el rumbo que se debe seguir para lograr el fin supremo de una empresa: maximizar ganancias con responsabilidad social y cuidando el medio ambiente.

En el presente artículo se analizan los principales determinantes de la gestión del capital humano en la industria de generación de energía eléctrica en Perú, la cual forma parte de un sector intensivo en capital físico en relación a sectores que son intensivos en mano de obra, como es la agricultura, la artesanía, entre otros.

1 Profesor Principal de la Facultad de Ingeniería Industrial, Departamento Académico de Producción y Gestión Industrial de la UNMSM. E-mail: nestor_sj@hotmail.com. 
Tal como se puede apreciar en la Figura N. ${ }^{\circ} 1$, las empresas de generación eléctrica producen energía utilizando como fuente de generación el agua, petróleo, gas, carbón, viento, entre otros; dicha energía es distribuida a través de empresas de transmisión, quienes a su vez la entregan a empresas distribuidoras o a usuarios finales, según la modalidad de venta aplicada por la empresa de generación. Las empresas distribuidoras comercializan energía básicamente a usuarios finales de menor consumo unitario, denominado consumo residencial y público; mientras que, los usuarios finales que requieran volúmenes importantes de energía, denominados grandes consumidores, como las empresas mineras y las industrias en general, son atendidos directamente por las empresas generadoras.

\section{MUESTRA DE EMPRESAS SELECCIONADAS}

Para el análisis de la gestión del capital humano se ha seleccionado a empresas de generación eléctrica de Perú, que durante el año 2010 aportaron la mayor producción de energía. Según información del Comité de Operaciones Económicas (COES) del Sistema Eléctrico Interconectado Nacional (SEIN), la producción de cuatro empresas (de un total de veintidós) explican prácticamente las dos terceras partes $(66,6 \%)$ de la producción total de energía eléctrica. Dichas empresas, para los fines del presente artículo, se identifican en la tabla $\mathrm{N}^{\circ} 1$.

Las empresas seleccionadas tecnológicamente no son comparables, dado que una central hidroeléctrica requiere un monto importante de inversión inicial y su costo de operación y mantenimiento (O\&M) es relativamente bajo, en relación a una central térmica, la cual requiere bajas inversiones en infraestructura pero sus costos de O\&M son altos. A pesar de ello, es interesante analizar, para fines ilustrativos, las similitudes y diferencias en la gestión del capital humano durante el periodo 2007-2010. La información utilizada es la publicada en la página web de la Superintendencia del Mercado de Valores (SMV), antes CONASEV, correspondiente a los estados financieros auditados (1) y la memoria anual de las cuatro empresas de la muestra (2).

En la Figura $\mathrm{N}^{\circ} 2$ se puede apreciar que no existe una relación directa entre el activo total y el número de trabajadores; ello se debería a las diferentes tecnologías utilizadas, la cantidad de las plantas o centros de producción de energía eléctrica y la antigüedad de las instalaciones de las empresas.

\section{Figura $\mathrm{N}^{\circ} 1$ : Sistema eléctrico nacional}

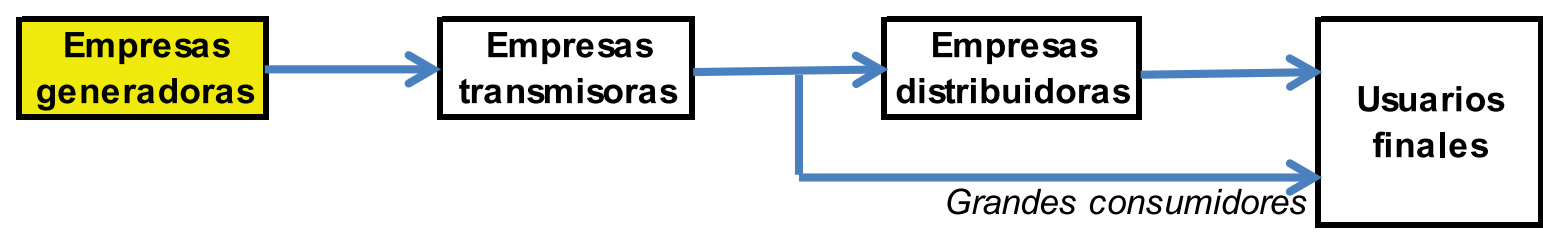

\begin{tabular}{|c|c|}
\hline \multicolumn{2}{|c|}{ Tabla N01: Identificación de empresas seleccionadas } \\
\hline Empresa A & $\begin{array}{l}\text { Empresa de menor tamaño o capacidad, de propiedad privada, que cuenta } \\
\text { |principalmente con centrales hidroeléctricas y en menor medida con centrales } \\
\text { térmicas a petróleo y a gas. }\end{array}$ \\
\hline Empresa B & $\begin{array}{l}\text { Es la empresa de mayor tamaño o capacidad de generación de la muestra, de } \\
\text { propiedad privada, con centrales hidroeléctricas y térmicas a gas y a petróleo. }\end{array}$ \\
\hline Empresa C & $\begin{array}{l}\text { Empresa de gran tamaño, menor a la capacidad de la Empresa B, de propiedad del } \\
\text { Estado, que posee el principal centro de generación hidroeléctrica del país. }\end{array}$ \\
\hline Empresa D & Empresa privada, de tamaño intermedio, principalmente con centrales térmicas a gas \\
& y a carbón y en menor medida centrales hidroeléctricas. \\
\hline Fuente: SMV. Elaboración propia.
\end{tabular}




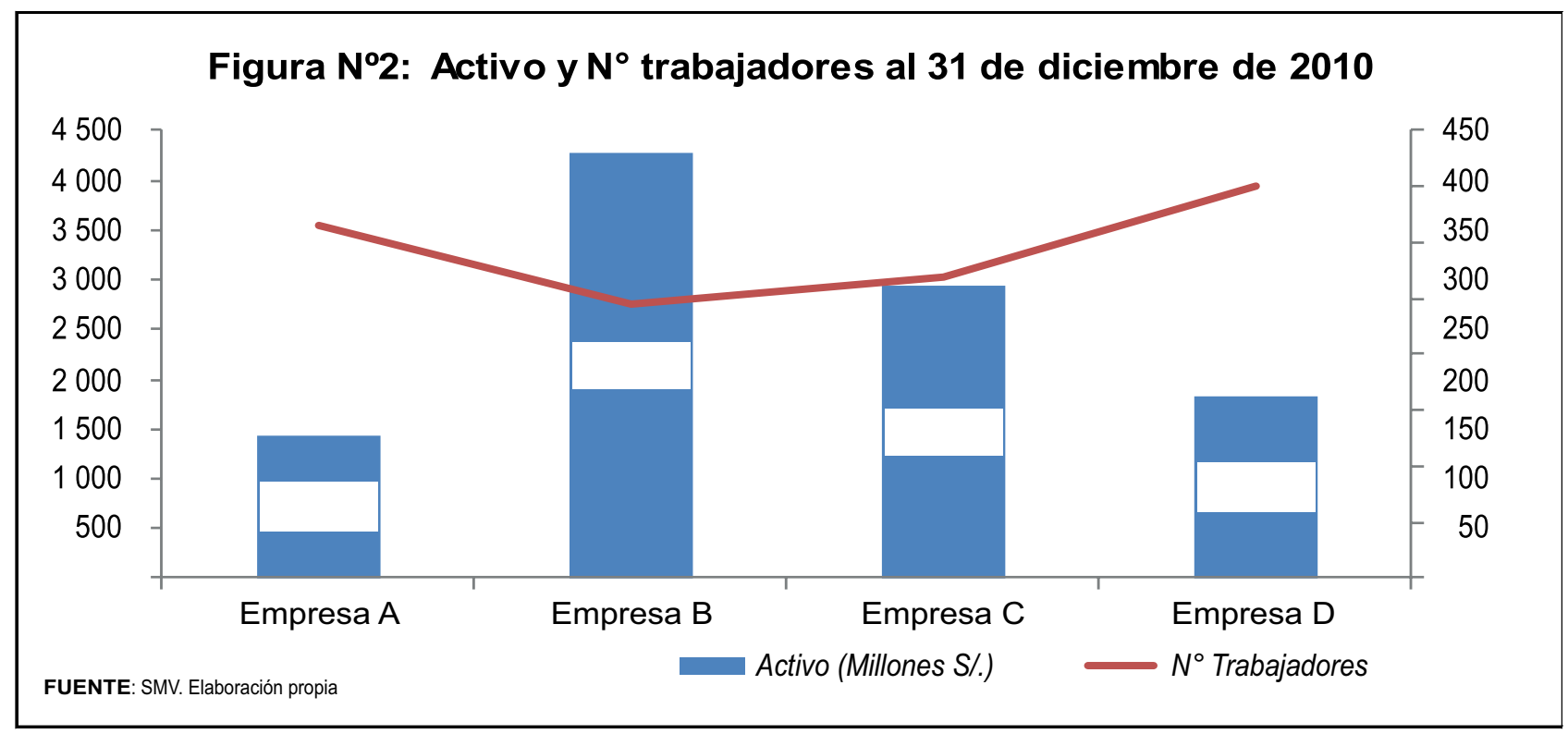

Al 31 de diciembre de 2010, el activo total que figura en el balance general (ahora posición financiera) de las empresas, que es la inversión neta registrada a la indicada fecha, varía entre 4,55 millones y 17,44 millones de nuevos soles por cada trabajador; ratio que corresponde a las Empresas $A$ y $B$, respectivamente. La Empresa $A$, que es la más pequeña, cuenta con tres centros de producción hidroeléctrica y ocho centros de producción térmica; en cambio la Empresa B, la más grande, posee siete centrales hidroeléctricas y dos centros de generación térmica. En la Empresa $B$ hay mayor capital físico comprometido por trabajador, es de tener en cuenta que las centrales hidroeléctricas son las que requieren mayores niveles de inversión en activos y dan empleo a la menor cantidad de trabajadores.

\section{ÍNDICES DE PRODUCTIVIDAD Y DE EFICIENCIA}

El capital humano debidamente gestionado contribuye en forma significativa en la mejora de la pro- ductividad y de la eficiencia de una empresa, en términos del valor generado y el gasto incurrido por cada trabajador, respectivamente.

a) Productividad

Una manera de expresar la productividad del trabajador, que es la relación entre el valor del resultado obtenido y el valor del insumo utilizado en dicho resultado, consiste en dividir el valor de la producción en términos del mercado eléctrico -representado por los ingresos por ventas-y el número de trabajadores de cada ejercicio fiscal.

De acuerdo a la Tabla N. ${ }^{\circ} 2$, en promedio durante el periodo 2007-2010, de acuerdo al estado de ganancias y pérdidas (ahora estado de resultados) las Empresas B y $C$ presentan los mayores niveles de productividad con un ingreso por ventas de $\mathrm{S} / .4,5$ millones y $\mathrm{S} / .4,4$ millones por trabajador, respectivamente.

\begin{tabular}{|c|r|r|r|r|}
\hline \multicolumn{5}{|c|}{ Tabla No2: Productividad del trabajador (S/./trabajador) } \\
\hline Año & Empresa A & Empresa B & Empresa C & Empresa D \\
\hline 2007 & 2012076 & 4681885 & 4472126 & 2351502 \\
2008 & 1749930 & 4746760 & 4629246 & 4013955 \\
2009 & 1330607 & 4429862 & 4629246 & 2853641 \\
2010 & 988962 & 4247209 & 3864293 & 3202669 \\
\hline Promedio & 1520394 & 4526429 & 4398727 & 3105442 \\
Desviación Estándar & 451987 & 230941 & 363907 & 699208 \\
\hline Fuente: SMV. Elaboración propia.
\end{tabular}


En el caso de la Empresa A, que es la de menor tamaño de la muestra, se observa un aumento en el personal empleado especialmente en los últimos dos años, pero a su vez presenta una disminución gradual en los ingresos por ventas, tal como se registra en la Figura N. ${ }^{\circ} 3$; lo que lugar a que la productividad del trabajador de esta empresa sea la más baja de la muestra.

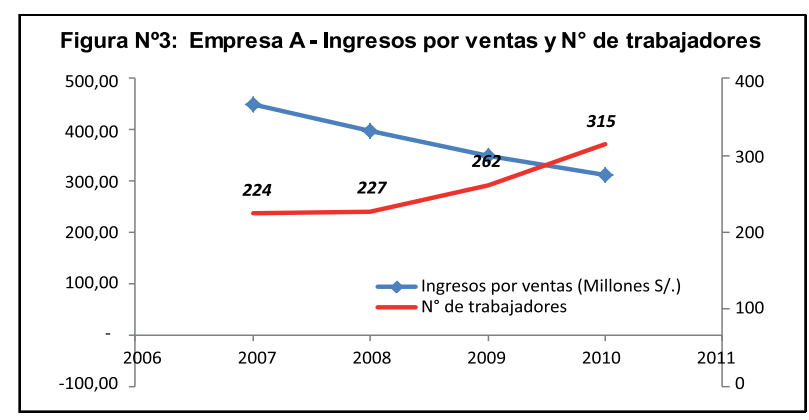

b) Eficiencia

El esfuerzo de la organización para obtener los ingresos por ventas se refleja en los costos de operación, los cuales están representados por la suma de los costos de producción o del servicio y los gastos de administración y de ventas; en dichos costos se incluyen a los recursos utilizados en el talento humano, que dan lugar a los gastos de personal.

\section{Participación de gastos de personal en los cos- tos de operación}

Según la Tabla $N .^{\circ} 3$, para las Empresas $B, C$ y D los gastos de personal en promedio representan alrededor del $6,4 \%$ de los costos de operación. En la Empresa A, dicha participación es elevada, valor que ha ido aumentado en los últimos dos años. Este efecto se debería a que los gastos de personal, excepto la participación de las utilidades, es una carga fija, la que ante una disminución del nivel de ingresos por ventas limita su optimización.

\begin{tabular}{|c|c|c|c|c|}
\hline \multicolumn{5}{|c|}{ Tabla N`3: Participación de gastos de personal en los } \\
costos de operación \\
\hline Año & Empresa A & Empresa B & Empresa C & Empresa D \\
\hline 2007 & $14,6 \%$ & $5,7 \%$ & $5,5 \%$ & $5,8 \%$ \\
2008 & $9,8 \%$ & $4,2 \%$ & $5,5 \%$ & $6,9 \%$ \\
2009 & $17,1 \%$ & $6,0 \%$ & $7,6 \%$ & $7,5 \%$ \\
2010 & $16,5 \%$ & $6,6 \%$ & $7,8 \%$ & $7,3 \%$ \\
\hline Promedio & $\mathbf{1 4 , 5 \%}$ & $\mathbf{5 , 6} \%$ & $\mathbf{6 , 6} \%$ & $\mathbf{6 , 9} \%$ \\
\hline \multicolumn{5}{|l}{} \\
\hline
\end{tabular}

\section{Gastos de personal por trabajador}

Este indicador es marcadamente diferente para las empresas seleccionadas. En promedio en las Empresas $\mathrm{B}$ y $\mathrm{C}$, las más grandes de la muestra, se observa el mayor gasto por cada trabajador al año, y cuya evolución se puede apreciar en la Tabla $\mathrm{N}^{\circ} 4$.

\begin{tabular}{|c|c|c|c|c|}
\hline \multicolumn{5}{|c|}{ Tabla N`4: Gastos de personal por trabajador (Nuevos soles / trabajador) } \\
\hline Año & Empresa A & Empresa B & Empresa C & Empresa D \\
\hline 2007 & 162393 & 196088 & 195358 & 89666 \\
2008 & 141859 & 156645 & 185765 & 182921 \\
2009 & 159248 & 173979 & 228458 & 154987 \\
2010 & 122584 & 202508 & 203885 & 171538 \\
\hline Promedio & $\mathbf{1 4 6 5 2 1}$ & $\mathbf{1 8 2} \mathbf{3 0 5}$ & $\mathbf{2 0 3 3 6 7}$ & $\mathbf{1 4 9} 778$ \\
\hline \multicolumn{5}{|l}{} \\
\hline
\end{tabular}

Un componente importante de los gastos de personal es la participación de los trabajadores de las utilidades de la empresa, la que para el sector eléctrico peruano equivale al $5 \%$ de la utilidad antes del pago del impuesto a la renta, cuyo importe anual se distribuye entre los trabajadores en planilla, el 50\% según los días laborados al año y el otro $50 \%$ en proporción al monto total de la remuneración. De acuerdo a la Figura $\mathrm{N}^{\circ} 4$, en las Empresas $C$ y $D$, que generan importantes ganancias netas, la participación de trabajadores de las utilidades explica una proporción significativa de los gastos de personal.

Además de la indicada participación de trabajadores de las utilidades, forman parte de los gastos de personal los siguientes conceptos: remuneraciones,

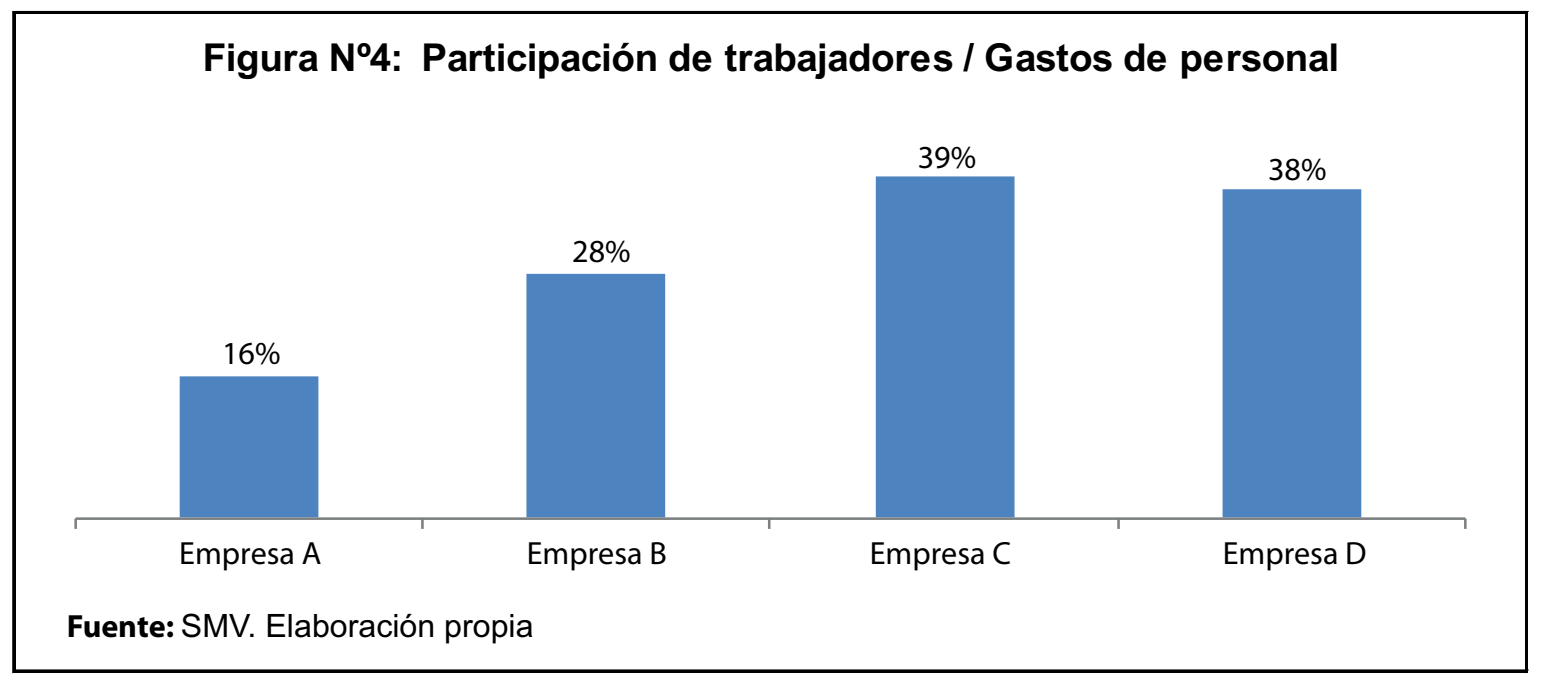


contribuciones sociales, vacaciones, bonificaciones, capacitaciónes, horas extra, entre otros.

\section{RESPONSABILIDAD SOCIAL INTERNA}

Cada empresa cuenta con mecanismos para motivar a sus trabajadores, buscando un adecuado clima laboral que propicie la mejora continua de la productividad. Es responsabilidad de la administración asumir un compromiso íntegro con su capital humano, que motive al trabajador y desarrolle su competencia para el mejor desempeño de sus funciones.

Una adecuada remuneración y atractivos montos de participación en las utilidades de la empresa que puedan percibir los trabajadores, está acompañada de otros mecanismos de gestión del talento huma- no en las empresas de generación eléctrica peruanas, entre los que destacan los siguientes:

\section{a) Capacitación}

La capacitación es un factor motivador que se presenta en las cuatro empresas seleccionadas. En su Memoria Anual, las Empresas B y C, indican horas de capacitación al año, además de información cualitativa y/o presupuesto ejecutado en capacitación como lo reportan las otras dos empresas.

Durante el periodo 2007-2010, las empresas B y C, dedicaron 63,3 y 37,6 horas de capacitación al año y por trabajador, respectivamente. En la tabla $\mathrm{N}^{\circ} 5$ se detallan las principales actividades de capacitación ejecutadas durante el periodo considerado para el análisis.

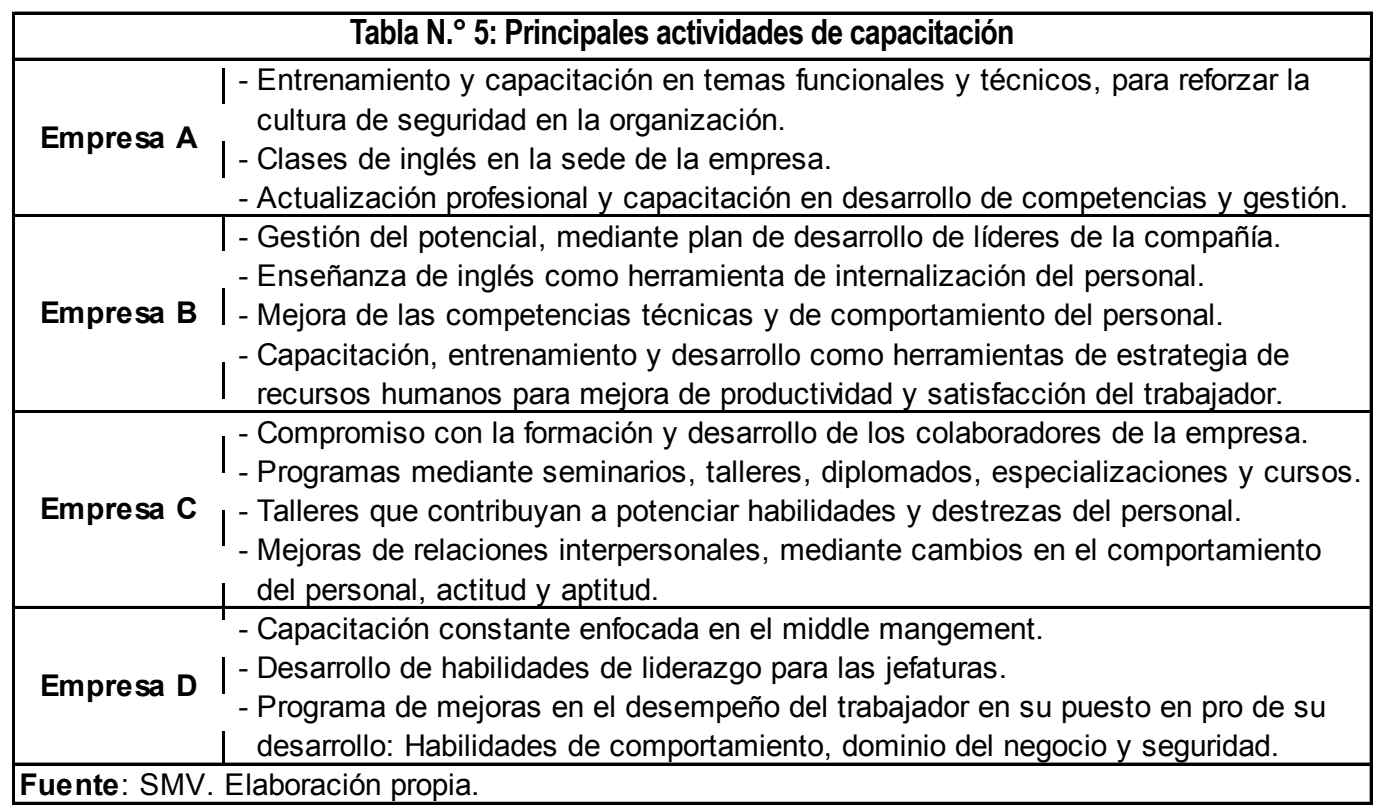

\section{b) Programas de reconocimiento}

Destaca el reconocimiento público del tiempo de servicios de los trabajadores, en reuniones trimestrales; asimismo, se consideran premios por comportamientos, rendimientos y conductas destacadas del trabajador.

En la Empresa A, cada cinco años de servicios, se entrega un cuadro de reconocimiento, además de un premio en función a la cantidad de quinquenios cumplidos, y cuenta con un programa de mejora continua que fomenta la implementación de proyectos que tengan por finalidad mejorar las operaciones de la empresa. La Empresa B, en el año 2010, implementó un sistema de evaluación denominado BARS que mide el comportamiento del personal con base a estándares de conductas determinados por el Grupo Empresarial a nivel mundial. En la Empresa $\mathrm{D}$ destaca el sistema de evaluación del desempeño con un enfoque en la productividad y la retroalimentación para la mejora continua.

La Empresa C no publica en su Memoria Anual su programa de reconocimiento de sus trabajadores.

\section{c) Clima laboral}

La mejora continua y el desarrollo de las competencias organizaciones son una constante, que incluye el desarrollo de iniciativas para generar un ambiente de trabajo de alta calidad donde los trabajadores tienen los recursos y el ambiente propicio para desarrollar sus labores; el reto para la administración es promover el buen clima laboral, además de fomentar una comunicación abierta entre los trabajadores.

La Empresa A, en el año 2008, por sexto año consecutivo fue premiada como una de las 30 mejores empresas para trabajar en Perú, según la encuesta 
que anualmente realiza el instituto Great Place to Work. En la Empresa B se promueve la gestión del potencial del personal profesional y de dirección; y cuenta con un plan de rotación que tiene por finalidad el fortalecer las habilidades del personal, enriquecer sus conocimientos, ampliar sus horizontes profesionales y compartir experiencias.

En las Empresas $C$ y $D$ destacan las buenas prácticas para reforzar la motivación, la confraternidad y el trabajo en equipo.

\section{d) Bienestar social}

Este beneficio se refleja en la importancia de equilibrar la vida laboral y personal de los trabajadores, buscando el bienestar del equipo humano que labora en la empresa.

La Empresa A ofrece beneficios como horarios de trabajo flexible, un día libre al año y eventos destinados a integrar a los trabajadores con sus familias. En las Empresas B y $C$ se realizan celebraciones emblemáticas, que comprende fiesta anual de confraternidad, concursos y la práctica de deportes; además, destaca el plan de prevención de la salud y temas afines, orientación médica personalizada y desarrollo de actividades culturales.

En la Empresa $D$ se difunden los diversos beneficios que ofrece la empresa a todos sus trabajado- res y la creación de valor en los usuarios que estos beneficios significan.

\section{e) Sindicatos}

Es compromiso de la administración de una empresa el respeto a los derechos laborales y al principio de libertad sindical, para que el sindicato de trabajadores se constituya en un colaborador importante de la gestión del capital humano y sea socio estratégico para la consecución de los objetivos de la empresa.

La Empresa B tiene un convenio colectivo con la organización sindical, con una vigencia de 5 años, que rige desde el año 2009; el $47 \%$ de su personal integra el sindicato. En la Empresa D, la tendencia es la suscripción del pliego de reclamos con el Sindicato de mayor plazo y en menos tiempo de negociación; el pliego suscrito en el año 2010 tiene una vigencia de dos años.

Las Empresas A y C no publican en su Memoria Anual las actividades que desarrollan con sus sindicatos de trabajadores.

\section{f) Otros beneficios}

Adicionalmente, en la Tabla N ${ }^{\circ} 6$ se detallan los diversos beneficios que perciben los trabajadores de la muestra analizada.

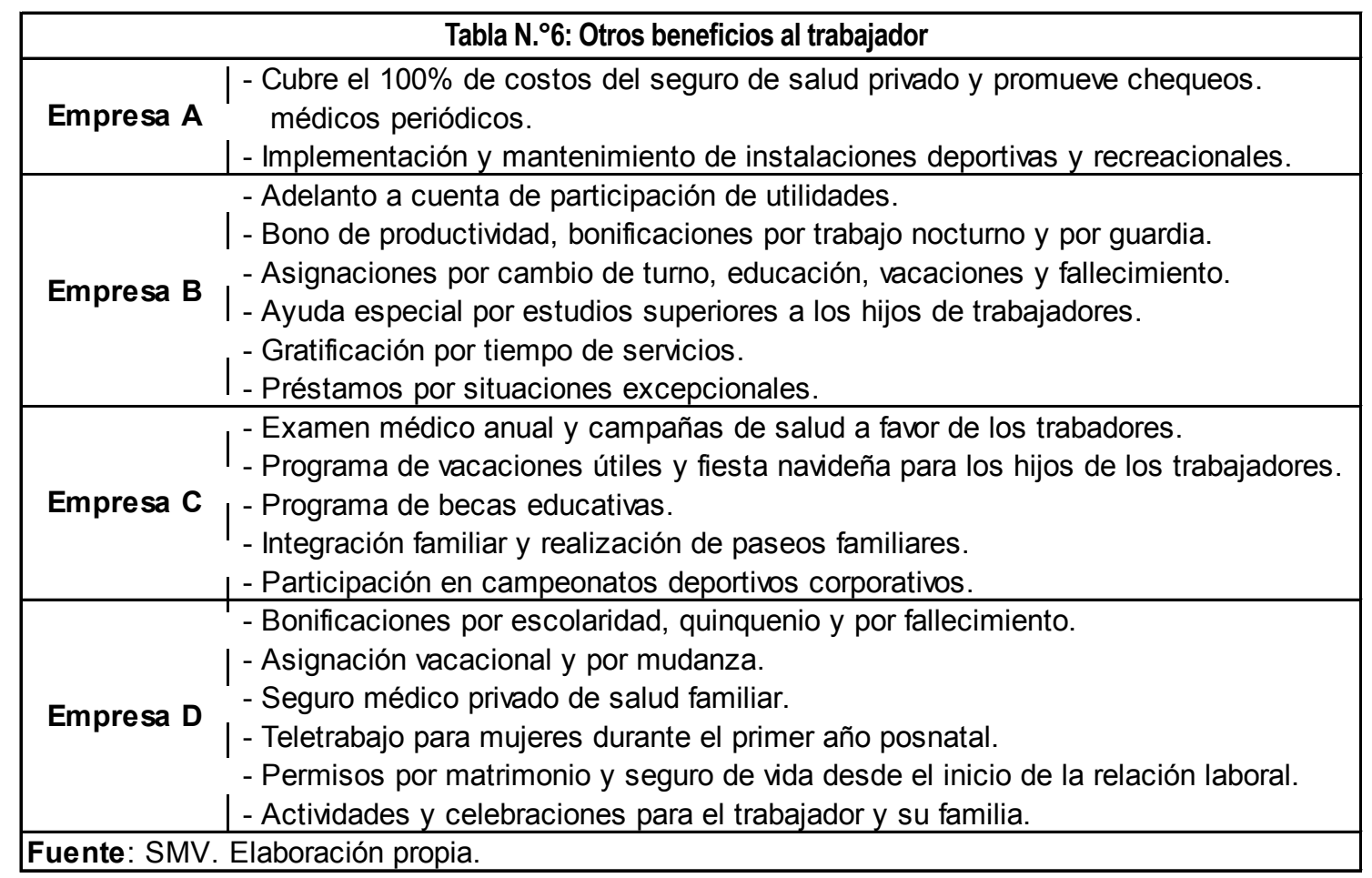




\section{CONCLUSIONES}

1. Los índices de eficiencia y productividad observados en el periodo 2007-2010 no son homogéneos, varían significativamente entre las empresas dependiendo de la tecnología utilizada en la generación de energía eléctrica y la cantidad de plantas o de centros de producción de cada empresa. La productividad anual promedio de cada trabajador se encuentra entre S/.1,5 millones y $S / .4,5$ millones de ingresos por ventas.

2. Un componente importante de la compensación económica que perciben los trabajadores es la participación en las utilidades anuales de la empresa, la cual en promedio varía entre $16 \%$ y $39 \%$ de gastos de personal.

3. La menor productividad laboral en términos de ingresos por ventas y la menor participación de las utilidades en gastos de personal se presenta en la Empresa $A$, que es la más pequeña de la muestra. La Empresa B, que es la más gran- de, registra la mayor productividad y la participación de utilidades representa el $28 \%$ de gastos de personal.

4. En las cuatro empresas de generación eléctrica analizadas destaca el esfuerzo de la administración de aplicar una política remunerativa competitiva, la que es complementada con beneficios muy atractivos para sus trabajadores, lo que contribuye a la mejora del clima laboral y a lograr resultados adecuados para la organización empresarial.

\section{REFERENCIAS BIBLIOGRÁFICAS}

[1] SMV (2011). Información financiera individual auditada anual de las empresas seleccionadas. http://www.smv.gob.pe/emisoras/emi_eeff_ menu.asp (visitado el 04-08-2011).

[2] SMV (2011). Memoria anual de las empresas seleccionadas. http://www.smv.gob.pe/emisoras/Emi_memoria.asp?p_codigo=115926 (visitado el 05-08-2011). 